\title{
Métodos parasitológicos aplicados em espécimes de solo e água em setores do município de Guaraí/TO
}

As parasitoses são consideradas um problema de saúde pública devido à deficiência no saneamento básico em diversas cidades brasileiras. Resultante disto, as pessoas contraem doenças parasitárias que, embora sejam de fácil tratamento, ainda não foram erradicadas. Além disso, as companhias de abastecimento de água e pesquisas ambientais não desenvolvem pesquisas parasitológicas, o que contribui para que a população continue leiga no assunto e vulnerável aos parasitos. Com base nisto, o objetivo deste estudo foi a aplicação de métodos parasitológicos na avaliação da contaminação da água e solo de alguns setores do município de Guaraí em busca de protozoários e helmintos. Para o desenvolvimento, foi realizado uma pesquisa de campo em amostras coletadas em alguns setores do município e pesquisa literária de caráter descritivo, qualitativo e exploratório, por meio de livros, artigos científicos, dissertação e monografia pesquisados nos sites Google Acadêmico e Scientific Electronic Library Online (SCIELO). Foi obtido também dados de resultados de exames parasitológicos de fezes positivos em dois laboratórios da cidade e informações sobre o saneamento básico e parasitoses no Brasil por meio dos sites do Departamento de Informática do SUS (DATASUS) e Trata Brasil. Para a análise da água foi realizado os métodos direto a fresco, sedimentação espontânea (Hoffmann) e centrifugo-sedimentação, a análise do solo foi através de Hoffmann e Rugai. Os principais parasitos encontrados na água foram cistos de Endolimax nana e Giardia lamblia e Trofozoíto de Ballantidium coli; no solo, as principais espécies identificadas foram Entamoeba coli e larva de Strongyloide stercoralis.

Palavras-chave: Parasitoses intestinais; Epidemiologia; Saneamento Básico.

\section{Occurrence of leprosy in Brazil and the main methods of laboratory diagnosis}

Parasitoses are considered a public health problem due to deficiency in basic sanitation in several Brazilian cities. As a result of this, people contract parasitic diseases that, although they are easy to treat, have not yet been eradicated. In addition, water supply companies and environmental research do not develop parasitological research, which contributes to the population remaining on the subject and vulnerable to parasites. Based on this, the objective of this study was the application of parasitological methods in the evaluation of the contamination of water and soil of some sectors of the municipality of Guarai in search of protozoa and helminths. For the development, a field research was carried out in samples collected in some sectors of the city and literary research of descriptive, qualitative and exploratory character, through books, scientific articles, dissertation and monograph searched in the Google Academic and Scientific Electronic Library Online sites (SCIELO). Data were also obtained from parasitological exams of positive feces in two laboratories in the city and information on basic sanitation and parasitoses in Brazil through the websites of the Department of Information Technology of SUS (DATASUS) and Trata Brasil. For the analysis of the water was carried out the direct methods to fresh, spontaneous sedimentation (Hoffmann) and centrifugal-sedimentation, the soil analysis was through Hoffmann and Rugai. The main parasites found in the water were cysts of Endolimax nana and Giardia lamblia and Ballantidium coli trophozoite; in the soil, the main species identified were Entamoeba coli and Strongyloide stercoralis larvae.

Keywords: Intestinal parasites; Epidemiology; Basic sanitation.

\section{Topic: Parasitologia}

Reviewed anonymously in the process of blind peer.

Naissa Paula Ferreira Martins

Faculdade Guaraí, Brasil

http://lattes.cnpq.br/1837736917778703

paulamartinsz1996@gmail.com

Walquiria Sidneu de Souza

Faculdade Guaraí, Brasil

http://lattes.cnpq.br/5955946582624813

walquiriasidneu15@hotmail.com

Mara Soares de Almeida Mota (iD

Faculdade Guaraí, Brasil

http://lattes.cnpq.br/3061777057739833

http://orcid.org/0000-0002-2068-3481

mara.almeida06@gmail.com
Received: 13/12/2017

Approved: 22/12/2017
Referencing this:

MARTINS, N. P. F.; SOUZA, W. S.; MOTA, M. S. A.. Métodos parasitológicos aplicados em espécimes de solo e água em setores do município de Guaraí/TO. Scire Salutis, v.7, n.2, p.32-41, 2017. DOI: http://doi.org/10.6008/SPC2236-9600.2017.002.0004 


\section{INTRODUÇÃO}

Parasitologia é a ciência que estuda o parasitismo, ou seja, qualquer relação ecológica entre indivíduos de espécies distintas na qual se observa não somente a associação intima e duradoura, mas também as dependências metabólicas em diferentes estágios, no presente artigo foram pesquisados protozoários e helmintos. O histórico da parasitologia no Brasil acompanha a trajetória da medicina tropical, por volta de 1860 os parasitos se tornaram os responsáveis por importantes doenças do homem e de animais (REY, 2013; MASCARINI, 2003).

Calcula-se que as infecções intestinais causadas por protozoários e helmintos acometem cerca de 3,5bi de pessoas, desenvolvendo enfermidades em torno de $450 \mathrm{mi}$ em diversos lugares do mundo, afetando principalmente as crianças. Diarreias, desnutrição, anemia, retardo no crescimento e cognitivo, irritabilidade, imunodepressão e complicações agudas são umas das morbidades decorrentes (BELO et al., 2012).

Os parasitos encontram-se dispersos em diversos ambientes, que são favorecidos em resposta ao saneamento inadequado, a má distribuição de água potável, além do crescimento acelerado da população, particularmente em países subdesenvolvidos, entretanto, há poucos estudos sobre a prevalência dos mesmos, sendo a maioria baseado em dados adquiridos através de bases populacionais mal definidas. Para tanto, não se tem uma noção precisa de quantidade de infecções gastrointestinais causadas por estes microrganismos (MELO et al., 2015).

Define-se investigação epidemiológica como uma pesquisa onde são analisadas doenças já notificadas, e a partir desses dados, é feito um estudo aprofundado dos locais com predisposição de contaminação das pessoas com determinada enfermidade para identificar o agente transmissor, as vias de transmissão e os possíveis alvos. O objetivo é reunir informações sobre a patologia para que possam ser tomadas providências a fim de minimizar o risco de novas ocorrências do mesmo caso. A transmissão e disseminação do agente etiológico das doenças dependem de um processo de interação entre o meio ambiente, o agente transmissor e o ser humano, ele pode ser transmitido por fonte única como a água, os alimentos, o ar, ou pode ser resultante de exposição simples ao agente ou exposições continuadas por um determinado período de tempo (NEVES, 2005).

As parasitoses intestinais são um dos melhores parâmetros da situação socioeconômica de uma população, as quais estão associadas a diferentes fatores, como, precárias condições sanitárias, poluição do solo e da água por fezes infectadas, consumo de alimentos e água contaminados, fatores socioculturais, convivência com animais, faixa etária do hospedeiro e o tipo de parasito infectante. Embora no Brasil algumas modificações venham ocorrendo em vista de melhores condições de vida da população, as parasitoses continuam sendo enfermidades endêmicas em diversas regiões do país, representando grave problema de saúde pública (BELO et al., 2012).

As parasitoses são prejudiciais à saúde de crianças e adultos, e por esse motivo precisam ser prevenidas e tratadas. A incidência de poliparasitoses crescem a cada dia, e sabe-se que a participação da população como objeto de pesquisa, pode implicar em diversas dificuldades burocráticas na busca de dados 
e informações para o combate as parasitoses. Na maioria das vezes são vistas como doenças brandas e no entanto, não recebem atenção necessária, o que acaba contribuindo para a falta de dados sobre a prevalência das mesmas, deixando a população leiga ao assunto e exposta aos riscos.

Sendo assim, a busca de larvas, cistos, oocistos e ovos no habitat destes parasitos, pode ser um estudo de significância, pois assim, a população terá conhecimento acerca da exposição em que se encontra em ambientes sem saneamento, podendo tomar partido de medidas que tornem possível o combate eficaz e a prevenção aos agravos à saúde acometidos pelas doenças parasitárias. O objetivo deste estudo foi a aplicação de métodos parasitológicos na avaliação da contaminação da água e solo de alguns setores do município de Guaraí em busca de formas evolutivas de protozoários e helmintos.

\section{MATERIAIS E MÉTODOS}

Este estudo foi desenvolvido com base em uma pesquisa de campo com amostras coletadas em alguns setores do município e pesquisa literária de caráter descritivo, qualitativo e exploratório. Para a pesquisa literária, foram utilizados livros disponíveis na biblioteca da Faculdade Guaraí, artigos científicos, dissertação e monografia, obtidos por meio dos sites Google Acadêmico e Scientific Electronic Library Online (SCIELO). Também foi realizado um levantamento de dados dos resultados de exames parasitológicos de fezes positivo em dois laboratórios da cidade, sendo um privado e outro público e informes dos sites do caderno de informações do Departamento de Informática do SUS (DATASUS) e Trata Brasil.

A pesquisa de campo foi feita durante os meses de junho a setembro de 2017 , através da coleta de amostras de água e solo dos setores, Serrinha, Universitário, Pestana, Setor Sul, Alvorada 2 e Piassara, e os dados literários foram obtidos de fevereiro a outubro de 2017. Toda a coleta foi realizada utilizando os Equipamentos de Proteção Individual - EPI, para evitar possíveis contaminações. Primeiramente foi feita a análise macroscópica do ambiente de coleta, onde foi perceptível que os locais em questão são carentes em saneamento. As amostras foram colocadas em um frasco estéril, identificadas por setor, numeradas por pontos e conservadas em temperatura ambiente até o dia da análise.

Os espécimes de solo, foram submetidos aos métodos de sedimentação espontânea - Hoffmann - e Rugai, sendo o primeiro analisado após $24 \mathrm{~h}$ para eficácia da sedimentação e o segundo após $1 \mathrm{~h}$ para observar se houve migração de Larvas. A água foi observada direto a fresco e depois de 24 h através da sedimentação espontânea e centrifugo-sedimentação. Foram confeccionadas 5 lâminas, onde 3 foram coradas com lugol e 2 sem o lugol e analisadas em microscópio óptico na lente de 10 e 40 vezes. Os parasitos encontrados foram fotografados e os resultados foram distribuídos em tabelas por setor e tipo de amostra, foi confeccionado gráficos utilizando todos os setores, para demonstração em porcentagem da prevalência de parasitos em diferentes amostras. 


\section{RESULTADOS E DISCUSSÃO}

Foram colhidas dez amostras de água e dez amostras de solo em diferentes setores do município, as quais foram analisadas durante o mês de junho, tendo continuidade nos meses de agosto a setembro para confirmação dos primeiros resultados obtidos. Distribuindo por nível de contaminação, o setor com o maior número de amostras positivas do solo, foi o Setor Universitário, seguido pelos setores Pestana e Serrinha, o setor Piassara foi o que apresentou menor prevalência de parasitos. O setor Alvorada 2 e setor Sul, tiveram amostras negativas para parasitos. 0 gráfico 1 apresenta a porcentagem dos setores com maior prevalência de parasitos no solo.

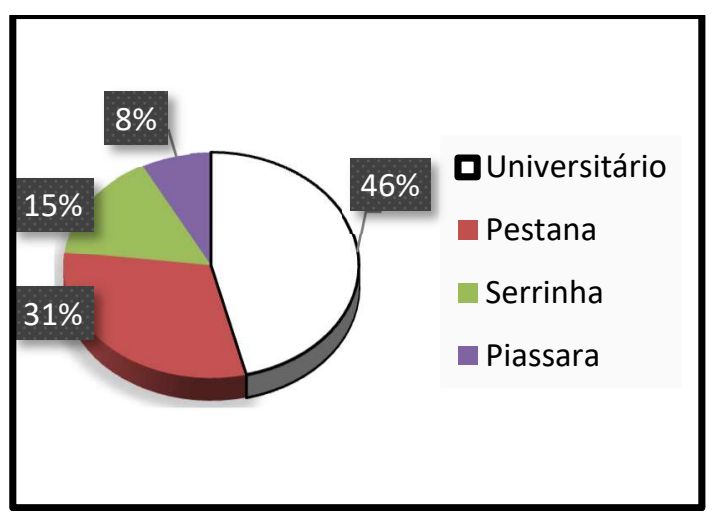

Gráfico 1: Porcentagem de amostra de solo positiva nos respectivos setores

Os métodos utilizados na pesquisa foram definidos baseando-se em um artigo que faz uma comparação com vários outros estudos, os quais evidenciam que a sedimentação espontânea foi realizada em $84,3 \%$ deles, considerando-se o baixo custo e praticidade, além da especificidade para determinados parasitos. $\mathrm{O}$ artigo apresenta os métodos parasitológicos que foram mais utilizados no período de 2008 a 2012, conforme representa o gráfico 2, o que comprova que o método de sedimentação espontânea é o mais realizado (HOLANDA et al., 2015).

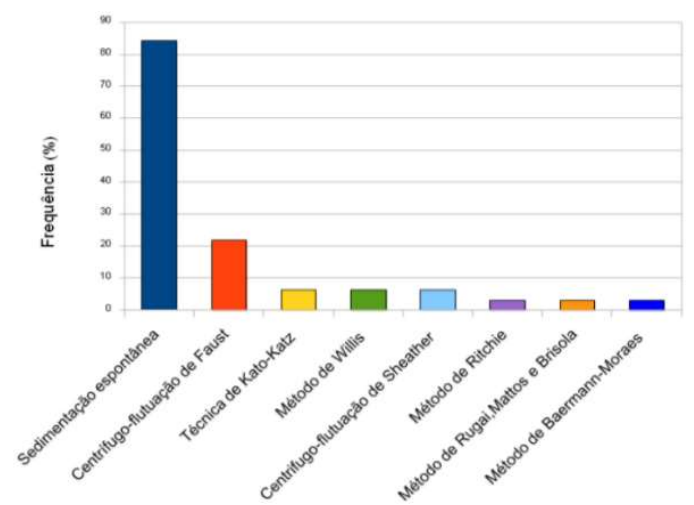

Gráfico 2: Métodos parasitológicos mais utilizados no Brasil entre o período de 2008 a 2012 Fonte: Holanda (2013).

Em outro estudo realizado em Palmas/TO, foram analisadas amostras de areia de cinco praias do município, através de três métodos parasitológicos: Hoffmann, Rugai e Faust. Foram detectadas 38 amostras contaminadas com parasito, dessas, obtiveram o seguinte resultado, pelo método de Hoffmann 27 amostras 
positivas, Faust, 9 e Rugai 2. Na pesquisa realizada em praças, do mesmo município, todas as amostras apresentaram positividade, 55,6\% foram analisadas pelo método de Hoffmann, 27,8\% Faust e 22\% pelo método de Rugai. Ou seja, foi comprovado por este estudo a eficácia e predominância do método de Hoffman tanto na análise da praia quanto na praça (SANTOS et al., 2010).

Dentre a metodologia empregada no presente estudo, a pesquisa de larvas através do método parasitológico de Rugai não foi satisfatória, pois nenhum resultado foi obtido. Em contrapartida, com o método de Hoffmann foi apropriado, no qual foram encontrados os protozoários e helmintos conforme descrito na tabela 1.

Tabela 1: Parasitos encontrados através dos métodos de Hoffmann em espécimes de solo.

\begin{tabular}{|l|l|}
\hline SETORES & PARASITOS \\
\hline UNIVERSITÁRIO & $\begin{array}{l}\text { Larva de Strongyloides stercoralis (Figura 1); ovo e larva de Ancilostomídeo (Figura 2 e 3); cisto de Giardia } \\
\text { lamblia (figura 4); trofozoíto de Ballantidium coli (Figura 5); cisto de Endolimax nana (Figura 6). }\end{array}$ \\
\hline PESTANA & Larva de Strongyloides stercoralis, cisto de Endolimax nana, Entamoeba coli e Entamoeba hystolitica. \\
\hline SERRINHA & Larva de Strongyloides stercoralis, Cisto de Entamoeba coli. \\
\hline PIASSARA & Entamoeba coli (Figura 7). \\
\hline
\end{tabular}

Figura 1: Larva de Stronyloides stercoralis.

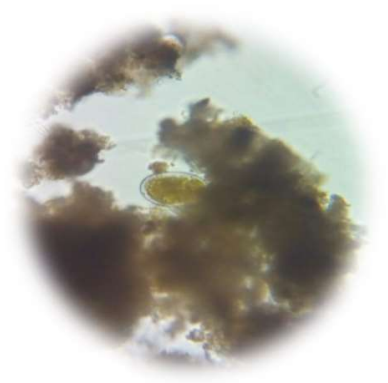

Figura 3: Ovo de Ancilostomídeo.

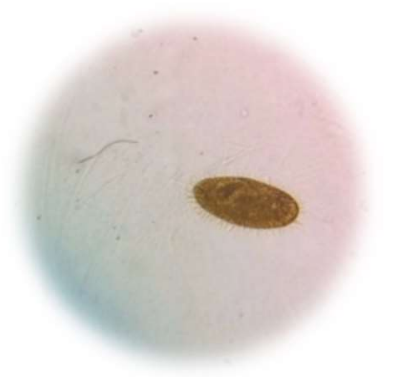

Figura 5: Trofozoíto de Ballantidium coli.

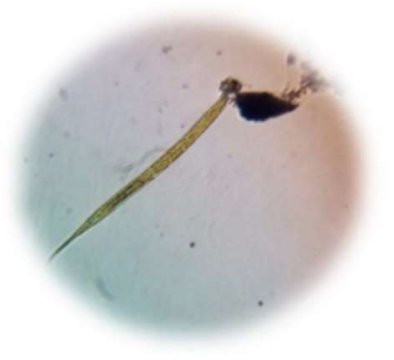

Figura 2: Larva de Ancilostomídeo

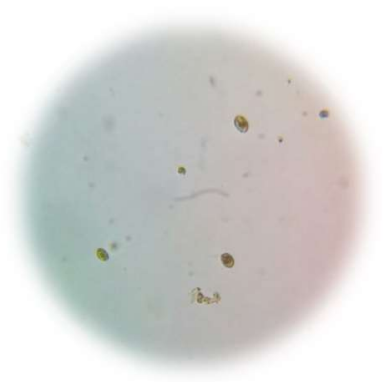

Figura 4: Cisto de Giardia lamblia.

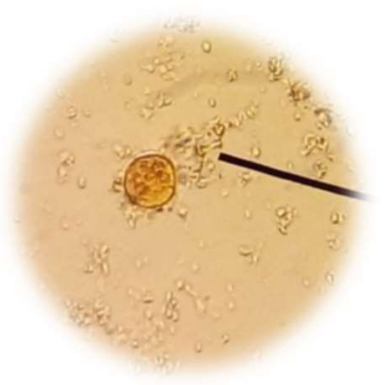

Figura 6: cisto Endolimax nana 
Figura 7: Cisto de Entamoeba coli.

Nos espécimes de água, apenas três amostras foram positivas, universitário, Pestana e Sul. Sendo utilizados três métodos, Hoffmann, Centrifugo-sedimentação e direto a fresco, enquanto Hoffmann não apresentou resultados positivos, os outros métodos apresentaram positividade. A porcentagem de amostras de água positiva por setor está apresentada no gráfico 3. Já a tabela 2 demonstra os setores com amostras de água positivas e quais parasitos foram encontrados.

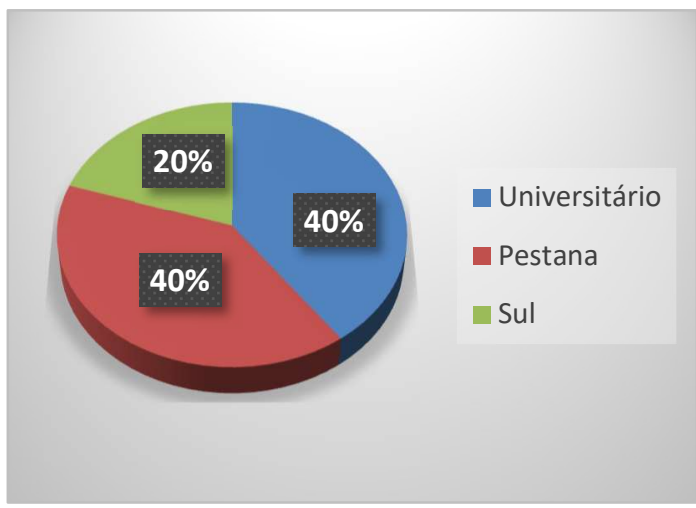

Gráfico 3: Porcentagem de amostra de água positiva nos respectivos setores.

Tabela 2: Parasitos em amostras de água nos respectivos setores.

\begin{tabular}{|l|l|}
\hline SETOR & PARASITOS \\
\hline UNIVERSITÁRIO & Cisto de Endolimax nana, Giardia lamblia. \\
\hline SUL & Ovo de Ancilostomídeo e cisto de Endolimax nana. \\
\hline PESTANA & Trofozoíto de Ballantidium coli. \\
\hline
\end{tabular}

É perceptível que, em comparação de amostras de solo e água, os setores Universitários e Pestana foram os que mais evidenciaram contaminação, tendo apresentando amostras positivas tanto em água quanto em solo. Apenas duas amostras tiveram contaminação monoparasitária, enquanto nas demais, observou-se contaminações simultâneas em uma mesma amostra, o que naturalmente é um risco especialmente para crianças que facilmente estão em contato com estes ambientes.

Todos os dados estão apresentados no gráfico 4, expostos segundo a prevalência de parasitos encontrados em todas as amostras, sendo possível observar que o nível maior de contaminação foi por Endolimax nana, em segundo lugar, Entamoeba coli e larvas de Strongyloides stercoralis, seguidos pela Giardia lamblia, Ballatidium coli e ovos de Ancilostomídeo, e em menor quantidade as Larvas de Ancilostomídeo. 


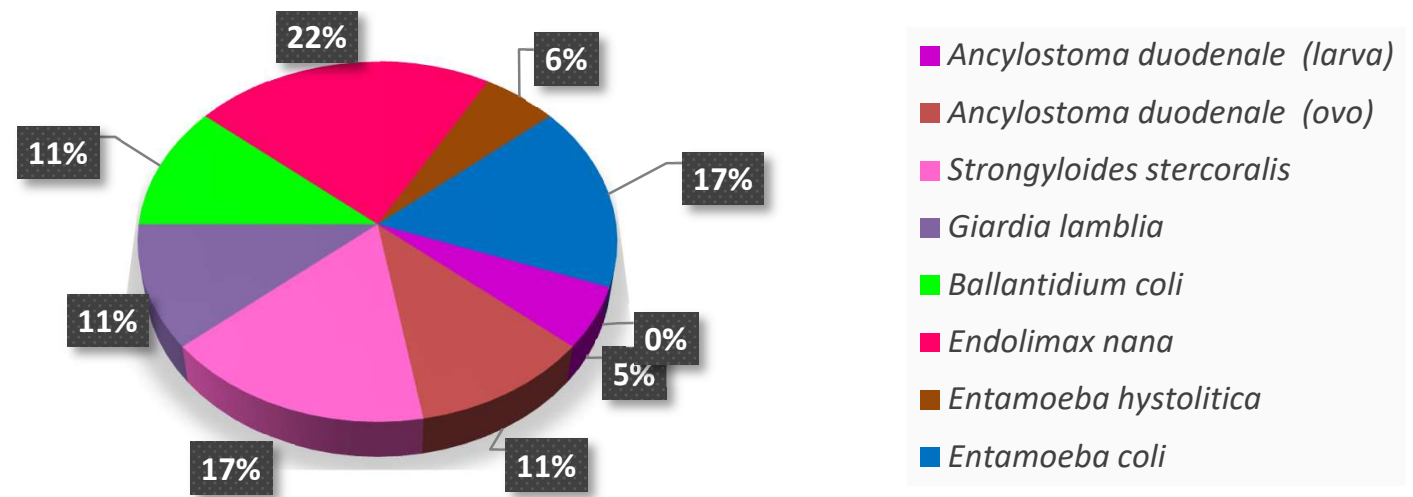

Gráfico 4: Porcentagem de parasitos em diferentes amostras de água e solo no município de Guaraí/TO nos meses de Junho e Setembro de 2017.

Estes resultados apresentam grande relevância para a população, pois como pôde observar, existem uma diversidade de parasitos que são de interesse clínico e desencadeiam doenças que levarão a quadros sintomáticos comuns e algumas complicações. Alguns autores afirmam que sintomas mais severos podem vir a ocorrer, tais como, pneumonite de hipersensibilidade em consequência da passagem das larvas na região pulmonar, outra consequência pode ser a disseminação maciça de larvas para outros órgãos durante o ciclo de autoinfecção do Strongyloides stercoralis, que poderá se unir a migração de enterobactérias para outro local que não sua biota normal, provocando sepse e meningite (MARTINS et al., 2008).

Embora os resultados positivos desta pesquisa tenham sido principalmente para Endolimax nana, dados coletados em um laboratório particular em Guaraí/TO, foi observado que a Entamoeba hystolitica e Giardia lamblia, estão entre os parasitos mais frequentes nas análises realizadas entre setembro de 2014 a setembro de 2017. O Gráfico 5 demonstra a quantidade de exame parasitológico de fezes positivos e negativos realizados entre os anos de 2014 a 2017 em um laboratório particular na cidade de Guaraí/TO.

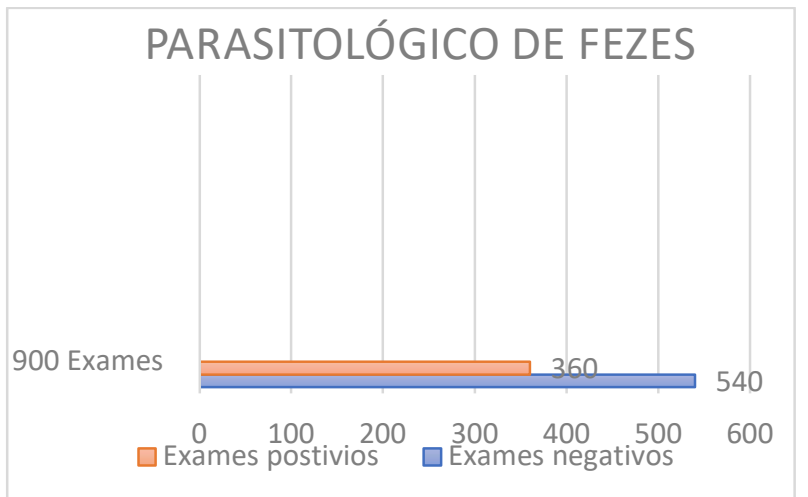

Gráfico 5: Quantidade de exames parasitológico de fezes positivos e negativos em um laboratório particular de Guaraí/TO.

Em 900 amostras, 360 foram positivas, o que confirma que as parasitoses ainda são muito frequentes. Em contrapartida, o Laboratório Municipal de Guaraí recebeu uma demanda maior de amostras positivas em um menor espaço de tempo, junho a setembro de 2017 , tendo em vista que os laboratórios municipais atendem um maior número de pacientes e liberam uma maior quantidade de laudos positivos.

No laboratório Municipal durante estes 4 meses, 467 amostras analisadas tiveram resultados positivos especialmente para Entamoebas coli e hystolitica e Giardia lamblia. Outro protozoário frequente 
foi Endolimax nana, os quais também foram muito observados nas amostras de solo e água do presente estudo. Em análises realizadas em praias e praças da capital Palmas/TO, ficou evidente que os parasitos mais observados em areias de praias foram, cisto de Ballantidium coli, ovo de Ancilostomídeo e oocisto de Toxoplasma gondii, seguidos por larvas de Strongyloides stercoralis, e outros. Nas análises de areia das praças, todas as amostras foram positivas para Strongyloides stercoralis, algumas positivas para ovos de Ancilostomideos, e as demais para Eimeria sp., Toxoplasma gondii, Ballantidium coli e outros (SANTOS, et al., 2010).

Em 2011, pesquisas realizadas em areia de praias da Paraíba, os parasitos mais encontrados foram, Endolimax nana e Entamoeba coli. De acordo com alguns autores, apesar de ambos os parasitos não terem importância clínica, por serem encontrados na flora normal do ser humano, identificá-los é importante porque são indicadores de contaminação fecal (OLIVEIRA FILHO et al., 2011).

Ao realizar análises microbiológicas e parasitológicas de amostras de água no município de Cascavel, a análise parasitológica não apresentou resultados positivos, em contrapartida, em uma pesquisa realizada em Distrito do Vale Verde, Minas Gerais, 37,5\% das amostras foram positivas para Giárdia lambia, Entamoeba histolytica e E.coli, em 25\% Isospora belli e em 12,5\% foi identificado os parasitos Balantidium coli, Strongyloides stercoralis e Entamoeba sp. Outro estudo relevante foi realizado em Aldeias de Guarani utilizando amostras de água tratada e bruta, ambas foram positivas para G. lamblia, Cryptosporidium spp. e Entamoeba histolytica (IARK et al., 2003; SILVA, 2016; BARBOSA et al., 2013).

Se comparadas, pode-se observar que na maioria das pesquisas, os resultados obtidos em amostras de solo e água contam com resultados semelhantes. Parasitos como Endolimax nana, Entamoeba coli, Entamoeba hystolitica, Ballantidium coli e Ancilostomídeo foram vistos com frequência em todos os estudos.

As parasitoses são enfermidades brandas que nem sempre são notificadas, o que dificulta a obtenção de dados anuais de sua incidência e prevalência, no entanto, alguns resultados baseados no município de Guaraí To, apontam que as parasitoses ainda se apresentam como fator de discussão, tendo apresentado 12,9\% das internações decorrentes de doenças parasitárias/infecciosas e 1,9\% foram causas de mortalidade envolvendo também os parasitos (BRASIL, 2009).

No Brasil, 14mi de ocorrências por afastamento do trabalho, foram por diarreias e vômito, levando trabalhadores a ficar longe de suas atividades em média de 3 dias, causando desconforto do mesmo, bem como para empresa, ademais, os custos do Sistema Único de Saúde foram de R\$355,71 por paciente. Se todas as pessoas tivessem acesso a saneamento básico e tratamento de esgoto, haveria uma redução de 74,6 mil internações, sendo $56 \%$ desta redução no Nordeste. A cada $R \$ 1,00$ investido em saneamento $R \$ 4,00$ seriam economizados na saúde (RIBEIRO, et al.,2010; TRATA BRASIL, 2014).

Em todo o país, apenas 50,3\% da população tem acesso a esgoto, sendo que, nas grandes cidades 3,5 bilhões de pessoas ainda o despejam em locais inapropriados mesmo recebendo coleta e tratamento do mesmo. Com base em estudos regionais, a pior situação está no norte do país, no qual só $16,42 \%$ recebe tratamento de esgoto. (BRASIL, 2015; NOGUEIRA, 2016). 
O controle e a prevenção de parasitoses podem ser feitos através da implantação de métodos simples, como sempre andar calçados, higienizar os alimentos e habituar-se a sempre lavar as mãos no decorrer do dia. Com esse intuito, foi criado o Movimento Brasil Sem Parasitoses, um projeto educacional, composto por vinte profissionais da Saúde que se deslocam por várias cidades do país, orientando a população a respeito da importância da adoção de bons hábitos de higiene e a conscientização da relevância do controle das verminoses.

\section{CONCLUSÃO}

O levantamento de dados sobre os parasitos encontrados no solo e na água são relevantes, pois por meio deles, a população adquire conhecimento de que os ambientes em que estão vivendo são habitat destes, e mesmo quando eles são assintomáticos, são indicadores de contaminação fecal, logo, a existência de outros parasitos também pode ocorrer. O presente artigo comprova em seus resultados a eficácia desse método de pesquisa, quando comparados os parasitos encontrados na analises de água e solo com os parasitos relatados pelos laboratórios da cidade. Visto a eficácia do estudo, o município de Guaraí consegue obter dados de parasitoses com menos burocracia e baixo custo. Fornecendo a população dados atuais e conscientizando sobre a importância do controle da doença e dos bons hábitos de higiene.

Durante a observação dos locais de coleta, notou-se que normalmente os locais eram poluídos, tinham fezes de animais e odores fortes, além disto, muitas crianças brincam descalças e estes fatores favorecem o surgimento e contaminação por parasitos. Sendo assim, a importância de adotar medidas profiláticas é deveras importante para evitar infecções parasitárias, sendo o uso de calçados, a redução da poluição por dejetos humanos, a higienização correta das mãos e alimentos algumas destas medidas. De nada adianta um local com acesso a saneamento se a própria população continuar com estes hábitos.

\section{REFERÊNCIAS}

BARBOSA, A. S.; UCHÔA, C. M. A.; SILVA, V. L.; DUARTE, A. N.; CONCEIÇÃO, N. F.; VIANNA, M. B.; FONSECA, A. B. M. RIBEIRO, M. V. M.; BASTOS, O. M. P.. Avaliação parasitológica da água de abastecimento e do solo peridomiciliar de Aldeias Guarani. Revista Instituto Adolfo Lutz, São Paulo, v.72, n.1, p.72-80, 2013.

BELO, V. S.; OLIVEIRA, R. B.; FERNANDES, P. C.; NASCIMENTO, B. W. L.; FERNANDES, F. V.; CASTRO, C. L. F.; SANTOS, W. B.; SILVA, E. S.. Fatores associados à ocorrência de parasitoses intestinais em uma população de crianças e adolescentes. Revista Paulista de Pediatria, São João DelRei, v.30, n.2, p.1-7, 2012.

BRASIL. Ministério das Cidades. Caderno de informações: distribuição do percentual das internações por grupo de causas e faixa etária. Brasília: Ministério das Cidades, 2009.

BRASIL. Ministério das Cidades. Índices médios segundo região geográfica e Brasil. Brasília: Ministério das Cidades, 2017.
HOLANDA, T. B.; VASCONCELOS, M. C.. Geo-Helmintos: Análise E Sua Relação Com Saneamento. Revista Brasileira de Geografia Médica e da Saúde, Uberlândia, v.11, n.20, p.1-11, 2015.

IARK, A. C.; IDE, C.; KONOPATZKI, E.; RODRIGUES, M. C.. Análise microbiológica e parasitológica da água. In: SEMINÁRIO NACIONAL ESTADOS E POLÍTICAS SOCIAIS NO BRASIL. Anais. Cascavel: Unioeste, 2003.

MARTINS, F. S. V.; CASTIÑEIRAS, T. M. P. P.. Parasitoses intestinais: Diagnóstico e Tratamento. Centro de Informação em Saúde para viajantes, Rio de Janeiro, p.1-19, 2008.

MASCARINI, L. M.. Uma abordagem histórica da trajetória da parasitologia. Ciência \& Saúde Coletiva, Botucatu, v.8, n.3, p.809-814, 2003.

MELO, A. R.; ERICEIRA, F. V.; OLIVEIRA, N. D.; ROCHA, J. R.; FIRMO, W. C. A.. Ocorrência de parasitos intestinais em laudos parasitológicos de fezes de um laboratório privado do 
município de Bacabal/MA. Enciclopédia Biosfera, Goiânia, v.11, n.21, p.1-11, 2015

NEVES, D. P.; MELO, A. L.; LINARD, P. M.; VITOR, R. W. A. Parasitologia humana. 11 ed. Rio de Janeiro: Atheneu, 2005.

NOGUEIRA, D. J.. Efeitos do fenantreno e alquilbenzenos lineares no desenvolvimento larval e na transcrição de genes em ostra do Pacífico Crassostrea gigas (Thunberg, 1789). Dissertação (Mestrado em Aquicultura) Universidade Federal de Santa Catarina, Florianópolis, 2016.

OLIVEIRA FILHO, A. A.; FERNANDES, H. M. B.; ALCÂNTARA, N D. F.; ASSIS, J. C. F.; FREITAS, F. I. S.. Frequência de enteroparasitas nas areias das praias da Paraíba. Revista da Universidade Estadual da Paraíba, v.6, n.2, p.108-113, 2011
REY, L.. Bases da Parasitologia médica. 3 ed. Rio de Janeiro: Guanabara Koogan, 2013.

RIBEIRO, J. W.; ROOK, J. M. S.. Saneamento Básico e Sua Relação Com o Meio Ambiente e a Saúde Pública.

Monografia (Especialização em Análise Ambiental) Universidade Federal de Juiz de Fora, Juiz de Fora, 2010

SANTOS, E. S; SOUZA, H.. Pesquisa de helmintos e protozoários em areia de praias e praças na cidade de Palmas, Tocantins. Palmas: 2010.

SILVA, D. A.. Análise microbiológica e parasitológica da água do Distrito de Vale Verde, Minas Gerais. Ipatinga: Faculdade Única Ipatinga, 2016. 